\title{
Significance of Random Bladder Biopsies in Non-Muscle Invasive Bladder Cancer
}

\author{
Masafumi Kumano Hideaki Miyake Yuzo Nakano Masato Fujisawa \\ Division of Urology, Kobe University Graduate School of Medicine, Kobe, Japan
}

\section{Key Words}

Non-muscle invasive bladder cancer • Random biopsy • Urinary cytology

\begin{abstract}
Background/Aims: To evaluate retrospectively the clinical outcome of random bladder biopsies in patients with non-muscle invasive bladder cancer (NMIBC) undergoing transurethral resection (TUR). Patients and Method: This study included 234 consecutive patients with NMIBC who underwent random biopsies from normal-appearing urothelium of the bladder, including the anterior wall, posterior wall, right wall, left wall, dome, trigone and/or prostatic urethra, during TUR. Result: Thirty-seven patients (15.8\%) were diagnosed by random biopsies as having urothelial cancer. Among several factors available prior to TUR, preoperative urinary cytology appeared to be independently related to the detection of urothelial cancer in random biopsies on multivariate analysis. Urinary cytology prior to TUR gave $50.0 \%$ sensitivity, $91.7 \%$ specificity, $56.8 \%$ positive predictive value and $89.3 \%$ negative predictive value for predicting the findings of the random biopsies. Conclusion: Biopsies of normal-appearing urothelium resulted in the additional detection of urothelial cancer in a definite proportion of NMIBC patients, and it remains difficult to find a reliable alternative to random biopsies. Collectively, these findings suggest that it would be beneficial to perform random biopsies as part of the routine management of NMIBC.
\end{abstract}

Copyright $\odot 2013$ S. Karger AG, Basel

\section{KARGER}

Fax +4161306 1234

E-Mail karger@karger.ch

www.karger.com
(C) 2013 S. Karger AG, Basel

1015-9770/13/0072-0057\$38.00/0

Accessible online at:

www.karger.com/cur

\section{Introduction}

Approximately $80 \%$ of newly diagnosed urothelial carcinomas of the bladder are non-muscle invasive tumors that are limited to the urothelium or infiltrate no deeper than the lamina propria. The standard initial treatment for patients with non-muscle invasive bladder cancer (NMIBC) has been transurethral resection (TUR), which is performed to remove the tumor completely, assess the need for additional therapy, and plan a suitable follow-up schedule [1]. Although patients with NMIBC generally achieve good prognosis, showing survival rates at 5 years $>80 \%$ [2], some cases with this category of tumor exhibit an unfavorable clinical course, showing aggressive biological features [3]. Such heterogeneous clinical outcomes of NMIBC could be explained, at least in part, by concomitant malignant disease, which can be detected by random bladder biopsies during TUR $[1,2]$.

To date, there have been few studies investigating the significance of random bladder biopsies for patients with NMIBC, and their findings on the indication of this procedure were controversial [4-10]. For example, van der Meijden et al. [4] reported that random bladder biopsies contributed to neither the staging nor the selection of adjuvant therapy following TUR of NMIBC, while May et al. [5] showed the strong impact of random biopsies on the decision to undertake post-TUR therapy for patients with NMIBC. Considering these findings, we retrospectively reviewed the clinical outcomes of 234 consecutive patients with NMIBC who underwent random biopsies

Hideaki Miyak

Division of Urology, Kobe University Graduate School of Medicine

7-5-1 Kusunoki-cho, Chuo-ku

JP-650-0017 Kobe (Japan)

E-Mail hideakimiyake@hotmail.com 
of normal-appearing mucosa of the bladder to determine appropriate candidates likely to benefit from this procedure.

\section{Patients and Methods}

This study included 234 consecutive patients with either initially diagnosed or first-relapsed bladder tumors who were treated at our institution between 2004 and 2011 and fulfilled the inclusion criteria as follows: intravesical visible tumors were completely removed by TUR; resected tumors were pathologically diagnosed as NMIBC (i.e., Ta or T1 tumor); and multiple random bladder biopsies were performed following TUR. Prior to TUR, the number of tumors and the size of the largest tumor were recorded. In these series, random bladder biopsies of normal-appearing urothelial mucosa were carried out with a cold punch in the selected intravesical sites, including the anterior wall, posterior wall, right wall, left wall, dome, trigone and/or prostatic urethra.

Pathological examinations of primary visible tumors and biopsy specimens were performed by a single pathologist according to the 2002 American Joint Committee on Cancer TNM classification system. Cytological examinations of voided urine samples obtained from all patients before TUR were performed used standard $\mathrm{Pa}$ panicolaou staining and were classified into class I to V, and patients with either class IV or class $V$ were regarded as positive for this examination.

All statistical analyses were performed using Statview 5.0 software (Abacus Concepts, Berkeley, CA, USA). Differences in several clinicopathological parameters according to the findings on random biopsies were compared using the unpaired t test, Chi-square test or Mann-Whitney U test. Forward stepwise logistic regression analysis was used to determine the significance of preoperatively available parameters as predictors of the findings of random biopsies. $\mathrm{P}$ values $<0.05$ were considered significant.

\section{Result}

Of the 234 patients, concomitant urothelial cancer was detected in $37(15.8 \%)$ by random biopsies, while there were no positive findings in the remaining 197 (84.2\%). Of malignant lesions detected by the random biopsies, 34 $(91.9 \%)$ and $3(8.1 \%)$ were pathologically diagnosed as carcinoma in situ (CIS) and Ta tumor, respectively. Ta- ble 1 presents clinicopathological characteristics according to the findings of the random biopsies. Concomitant urothelial cancer was shown to be significantly likely to be detected by the random biopsies in patients with positive urinary cytology before TUR and those with multiple, T1 or Grade 3 tumors, despite the lack of significant impacts of the remaining factors on the outcomes of the random biopsies.

To identify preoperatively available parameters for predicting the probability of positive findings of the random biopsies, univariate and multivariate analyses were performed using the logistic regression model. As shown in table 2, univariate analysis identified preoperative findings of urinary cytology and multiplicity of visible tumors as significant predictors of the outcomes of the random biopsies. Furthermore, only preoperative findings of urinary cytology appeared to be independently associated with the probability of positive findings of the random biopsies. In fact, urothelial cancer was detected by the random biopsies in $16(8.3 \%)$ of the 192 patients negative for urinary cytological examinations, whereas $21(50.0 \%)$ of the 42 with positive findings of urinary cytology were diagnosed as having concomitant carcinoma by the random biopsies. That is, preoperative urinary cytology resulted in $50.0 \%$ sensitivity, $91.7 \%$ specificity, $56.8 \%$ positive predictive value and $89.3 \%$ negative predictive value for predicting the findings of the random biopsies.

\section{Discussion}

One of the most important features of urothelial cancer is the formation of tumors in multiple foci throughout the entire urinary tract, synchronously and/or metachronously. In fact, intravesical recurrence following TUR has been reported in 30 to $80 \%$ of patients with NMIBC, which could be partially explained by the presence of malignant lesions with normal appearance at the time of TUR $[1,2]$. To detect such concomitant urothelial cancers during TUR of NMIBC, random biopsies targeting normal-looking urothelial mucosa have been performed. However, there have been few studies of large series evaluating the significance of random bladder biopsies, and they have presented conflicting conclusions [4, 6, 7]. Against this background, the routine performance of random bladder biopsies for all patients with NMIBC is not recommended in the major guidelines, such as those by the European Association of Urology (EAU) and the National Comprehensive Cancer Network [11, 12], and 
Table 1. Characteristics of the included patients according to random biopsy

\begin{tabular}{|c|c|c|c|c|}
\hline & Overall & & Idom biopsy & \\
\hline & & Positive, $\mathrm{n}=37$ & Negative, $\mathrm{n}=197$ & $\mathrm{p}$ \\
\hline Age & $70 \pm 10$ & $71 \pm 8.8$ & $70 \pm 10$ & 0.44 \\
\hline Gender & & & & 0.94 \\
\hline Male & $197(84.2)$ & $31(83.3)$ & $166(84.3)$ & \\
\hline Female & $37(15.8)$ & $6(16.2)$ & $31(15.7)$ & \\
\hline Past history of UUTC & & & & 0.23 \\
\hline Positive & $25(10.7)$ & $6(16.2)$ & $17(8.6)$ & \\
\hline Negative & $209(89.3)$ & $31(83.8)$ & $180(91.4)$ & \\
\hline Urinary cytology before TUR & & & & $<0.001$ \\
\hline Class I, II or III & $174(74.4)$ & $16(43.2)$ & $175(88.8)$ & \\
\hline Class IV or V & $60(25.6)$ & $21(56.8)$ & $22(11.2)$ & \\
\hline Number of tumors & & & & 0.029 \\
\hline Solitary & $127(54.3)$ & $14(37.8)$ & $113(57.4)$ & \\
\hline Multiple & $107(45.7)$ & $23(62.2)$ & $84(42.6)$ & \\
\hline Tumor diameter, $\mathrm{cm}$ & & & & 0.067 \\
\hline$<3$ & $207(88.5)$ & $36(97.3)$ & $171(86.8)$ & \\
\hline 3 & $27(11.5)$ & $1(2.7)$ & $26(13.2)$ & \\
\hline Pothological stage & & & & 0.013 \\
\hline $\mathrm{Ta}$ & $177(74.4)$ & $22(59.5)$ & 155 (78.7) & \\
\hline $\mathrm{T} 1$ & $57(25.6)$ & $15(40.5)$ & $42(21.3)$ & \\
\hline Pathological grade & & & & $<0.001$ \\
\hline Grade 1 or 2 & $175(74.8)$ & $14(37.8)$ & $161(81.7)$ & \\
\hline Grade 3 & $59(25.2)$ & $23(62.2)$ & $36(18.3)$ & \\
\hline
\end{tabular}

Table 2. Univariate and multivariate analyses of factors associated with random biopsy

\begin{tabular}{|c|c|c|c|c|}
\hline & \multicolumn{2}{|c|}{ Univariate analysis } & \multicolumn{2}{|c|}{ Multivariate analysis } \\
\hline Sex (male vs. female) & 0.84 & 0.72 & 0.89 & 0.82 \\
\hline Past history of UUTC (negative vs. positive) & 1.91 & 0.2 & 1.59 & 0.42 \\
\hline Urinary cytology before TUR (class I, II or III vs. IV or V) & 11 & $<0.001$ & 9.57 & $<0.001$ \\
\hline
\end{tabular}

UUTC $=$ Upper urinary tract cancer.

thus the indications of random biopsies for patients with NMIBC remain controversial. Therefore, in this study, we retrospectively reviewed our experience of random bladder biopsies for patients with NMIBC undergoing TUR in order to identify the cohorts who are likely to benefit from this procedure.
In this series, urothelial cancer was detected by random bladder biopsies in $15.8 \%$ of the included patients. Of the 100 patients who were diagnosed as low risk NMIBC, including low grade, small size and solitary tumors, concomitant urothelial cancer was detected in $10(10 \%)$ patients by the random biopsies. To date, vari- 
ous proportions of patients showing positive findings of random bladder biopsies have been reported [4-10]. For example, May et al. [5] reported that $12.4 \%$ of patients with high- or intermediate-risk NMIBC had urothelial cancer in random biopsies, while in a study conducted by the European Organization for Research and Treatment of Cancer (EORTC), there were no abnormal findings of the random biopsies in $95.6 \%$ of patients with low-risk NMIBC and $88.4 \%$ of patients with high- and intermediate-risk NMIBC [4]. In addition, Hara et al. [10] reported that the incidence of positive findings of random biopsies for high- and intermediate-risk NMIBC was approximately 4 times as high as that in the EORTC study. These inconsistent detection rates could be explained by several factors, such as different indications of the random biopsies and variation in the number of biopsy cores among these studies. Therefore, it is necessary to collect the data of random biopsies carried out according to a strictly settled protocol in order to draw a definitive conclusion on this issue.

In this series, more than $90 \%$ of urothelial cancers detected by random biopsies were diagnosed as CIS, the most dominant risk factor for progression to muscle invasive disease, which should be intensively treated based on a strategy different from those of other NMIBCs [13]. This finding suggests that approximately one-sixth of patients with Ta or T1 tumors appeared to have concomitant CIS detectable by random bladder biopsies, which forced changes in the postoperative treatment as well as follow-up schedule in these patients. Furthermore, such an early diagnosis of CIS by random biopsies and optimal postoperative therapy, such as intravesical instillation of Bacillus Calmette-Guérin, may significantly contribute to improve the prognosis of patients with concomitant CIS, since there was no significant difference in the disease recurrence following TUR according to the outcome of the random bladder biopsies (data not shown).

We subsequently analyzed the association between several clinicopathological factors and the findings of the random biopsies, and found that patients with positive urinary cytology and those with multiple, T1 or Grade 3 tumors are likely to have concomitant urothelial cancers in normal-looking urothelium. To identify powerful predictive factors for the findings of the random biopsies, we further evaluated the values of preoperatively available parameters using logistic regression analyses, and preoperative findings of urinary cytology were shown to be independently associated with the probability of positive findings of the random biopsies. Hara et al. [10] previously reported a finding similar to that of this study; that is, dominant positive cytology was independently associated with the presence of concomitant CIS in patients with NMIBC. Although these findings suggest the usefulness of preoperative urinary cytology for predicting the findings of random biopsies, it remains unknown whether urinary cytology could be an alternative to random biopsies, even in a limited cohort of NMIBC with special characteristics. In this series, preoperative urinary cytology achieved $50.0 \%$ sensitivity and $91.7 \%$ specificity for predicting the findings of the random biopsies, indicating that concomitant urothelial cancer was detected in $8.3 \%$ of patients with negative urinary cytology by random biopsies. Considering these findings, the routine performance of random bladder biopsies for patients with NMIBC during TUR is recommended.

It has been well recognized that the execution of bladder biopsies is associated with some risks, including bleeding, perforation, infection and cancer dissemination [10]; therefore, it is necessary to develop novel strategies to avoid this procedure. One strategy is to accumulate a much larger number of patients with NMIBC undergoing random bladder biopsies prospectively and to identify predictive parameters with acceptable sensitivity as well as specificity in each group classified according to the EORTC risk tables [13]. Another approach is to introduce novel diagnostic modalities for concomitant urothelial cancer, such as fluorescence cystoscopy [14]. However, the substantial costs of using these devices present an obstacle to their widespread use in less developed countries.

In conclusion, the random bladder biopsies detected concomitant urothelial cancer in $15.8 \%$ of patients undergoing TUR for NMIBC, and the majority of the concomitant malignant tumors were pathologically diagnosed as CIS. Moreover, preoperative findings of urinary cytology appeared to be independently associated with the outcome of the random biopsies. However, urinary cytology resulted in $50.0 \%$ sensitivity and $91.7 \%$ specificity for predicting the presence of concomitant urothelial cancer. Collectively, the routine performance of random bladder biopsies during TUR of the NMIBC is recommended in order to avoid misdiagnosing concomitant urothelial cancer, particularly biologically aggressive CIS. 


\section{References}

1 Dalbagni G: The management of superficial bladder cancer. Nat Clin Pract Urol 2007;4: 254-260.

2 Nargund VH, Tanabalan CK, Kabir MN: Management of non-muscle-invasive (superficial) bladder cancer. Semin Oncol 2012;39: 559-572.

-3 van Rhijn BW, Burger M, Lotan Y, Solsona E, Stief CG, Sylvester RJ, Witjes JA, Zlotta AR: Recurrence and progression of disease in non-muscle-invasive bladder cancer: from epidemiology to treatment strategy. Eur Urol 2009;56:430-442.

-4 van der Meijden A, Oosterlinck W, Brausi M, Kurth KH, Sylvester R, de Balincourt C: Significance of bladder biopsies in Ta,T1 bladder tumors: a report from the EORTC Genito-Urinary Tract Cancer Cooperative Group. EORTC-GU Group Superficial Bladder Committee. Eur Urol 1999;35:267-271.

5 May F, Treiber U, Hartung R, Schwaibold H: Significance of random bladder biopsies in superficial bladder cancer. Eur Urol 2003;44: $47-50$.
6 Kiemeney LA, Witjes JA, Heijbroek RP, Koper NP, Verbeek AL, Debruyne FM: Should random urothelial biopsies be taken from patients with primary superficial bladder cancer? A decision analysis. Members of the Dutch South-East Co-Operative Urological Group. Br J Urol 1994;73:164-171.

7 Millan-Rodriguez F, Chechile-Toniolo G, Salvador-Bayarri J, Palou J, Vicente-Rodriguez J: Multivariate analysis of the prognostic factors of primary superficial bladder cancer. J Urol 2000;163:73-78.

8 Mufti GR, Singh M: Value of random mucosal biopsies in the management of superficial bladder cancer. Eur Urol 1992;22:288-293.

$>9$ Fujimoto N, Harada S, Terado M, Sato H, Matsumoto T: Multiple biopsies of normal-looking urothelium in patients with superficial bladder cancer: Are they necessary? Int J Urol 2003;10:631-635.

10 Hara T, Takahashi M, Gondo T, Nagao K, Ohmi C, Sakano S, Naito K, Matsuyama H : Discrepancies between cytology, cystoscopy and biopsy in bladder cancer detection after Bacille Calmette-Guerin intravesical therapy. Int J Urol 2009;16:192-195.
11 Babjuk M, Oosterlinck W, Sylvester R, Kaasinen E, Bohle A, Palou-Redorta J, Roupret M: EAU guidelines on non-muscle-invasive urothelial carcinoma of the bladder, the 2011 update. Eur Urol 2011;59:997-1008.

12 Brausi M, Witjes JA, Lamm D, Persad R, Palou J, Colombel M, Buckley R, Soloway M, Akaza H, Böhle A: A review of current guidelines and best practice recommendations for the management of nonmuscle invasive bladder cancer by the International Bladder Cancer Group. J Urol 2011;186:2158-2167.

13 Sylvester RJ, van der Meijden AP, Oosterlinck W, Witjes JA, Bouffioux C, Denis L, Newling DW, Kurth K: Predicting recurrence and progression in individual patients with stage Ta $\mathrm{T} 1$ bladder cancer using EORTC risk tables: a combined analysis of 2596 patients from seven EORTC trials. Eur Urol 2006;49: 466-475.

14 Fradet Y, Grossman HB, Gomella L, Lerner S, Cookson M, Albala D, Droller MJ; PC B302/01 Study Group: A comparison of hexaminolevulinate fluorescence cystoscopy and white light cystoscopy for the detection of carcinoma in situ in patients with bladder cancer: a phase III, multicenter study. J Urol 2007;178:68-73. 